\title{
ATAQUE ACELERADO DE ROCAS ÍGNEAS EN SOLUCIÓN DE SULFATOS DE SODIO. CASO DE ESTUDIO: TLALPUJAHUA MICHOACÁN, MÉXICO
}

\author{
D. GARCÍA-ROMERO, H. L CHÁVEZ-GARCÍA, J. C. GARCÍA-PERDOMO, \\ N. DÍAZ-GONZÁLEZ, J. A. PÉREZ-VELASCO
}

Universidad Michoacana de San Nicolás de Hidalgo, Facultad de Ingeniería Civil, Laboratorio de Materiales Ing. Luis

Silva Ruelas. Avenida Francisco J. Múgica, sin número, Ciudad Universitaria, C.P. 58030, Morelia Michoacán, México.

\begin{abstract}
RESUMEN
Evaluar el comportamiento de rocas ígneas simulando condiciones de exposición a sales en ambientes marinos, provocando deterioro prematuro. 3 distintos ejemplares de roca R (Rosa), G (Gris) y CO (café oscuro). Cada una fue cortada en 30 cubos de $5 \times 5 \times 5 \mathrm{~cm}$ se identificaron. Preparamos solución de sulfato de sodio según la NMX-C-075-ONNCCE-2006. Secamos muestras hasta masa constante y sumergimos cada una durante 18 horas, las sacamos y realizamos pruebas de masa húmeda, masa sumergida, Resistividad Eléctrica (RE), Densidad, velocidad de pulso Ultrasónico (VPU) y Compresión Simple. Durante 5 ciclos. La muestra R obtuvo menor pérdida de masa y en cada ciclo obtuvo mejores resultados comparado con las otras. Esperamos esta investigación contribuya a la conservación y construcción de edificaciones y monumentos.
\end{abstract}

Palabras clave: Roca; Sulfatos; Deterioro; Cubos.

\begin{abstract}
Evaluate behavior of igneous rocks simulating salt expisure condtions in marine enviroments, inducing premature deterioration. 3 differents specimens R(Pink), G (Gray) and CO (Dark Brown). Each one was cut into 30 cubes of $5 \times 5 \times 5 \mathrm{~cm}$ and marked. Prepared sodium sulphate solution according to NMX-C075-ONNCCE-2006. We dry each specimen until constant mass and submerge for 18 hours, remove and prove wet mass test, submerged mass, Electrical Resistivity (RE), Density, Ultrasonic PulseVelocity (o Rate??) (VPU) and Simple Compression. During 5 cycles. The specimen R had the lower mass loss and each cycle had better results compared to the others. Hoping that this research contributes at buildings and monuments conservation an construction.
\end{abstract}

Keywords: Rock; Sulphate; deterioration; Cubes.

\section{RESUMO}

Avaliar o comportamento de rochas ígneas simulando condições de exposição a sais em ambientes marinhos, causando deterioração prematura. 3 diferentes rochas R (Rosa), G (Cinza) e CO (marrom escuro). Cada um foi cortado em 30 cubos de $5 \times 5 \times 5 \mathrm{~cm}$ foram identificados. Preparamos solução de sulfato de sódio de acordo com NMX-C-075-ONNCCE-2006. Secamos as amostras até uma massa constante e submergimos cada uma delas por 18 horas, removemos e realizamos testes de massa úmida, massa submersa, Resistividade Elétrica (RE), Densidade, Taxa de Pulso Ultrassônico (VPU) e Compressão Simples. Durante 5 ciclos. A amostra R obteve menor perda de massa e em cada ciclo obteve melhores resultados em comparação aos demais. Esperamos que esta pesquisa contribua para a conservação e construção de edifícios e monumentos.

Palavras-chave: Rock; Sulfatos; Deterioração; Cubos. 


\section{INTRODUCCIÓN}

Una cantera es un espacio donde se extraen materiales pétreos, generalmente a cielo abierto, no obstante, en México el nombre también se le da a un tipo de roca volcánica ornamental utilizada en la construcción cuyas características principales son su fácil labrado, su porosidad, y su variedad de estas según su origen. Este tipo de roca se ha utilizado antes, durante y después de la época colonial, y es un símbolo arquitectónico de varios sitios a lo largo del país.

La cantera usada para este experimento fue extraída Tlalpujahua municipio del Estado de Michoacán de Ocampo, una de las principales ciudades mineras durante la Colonia. La explotación de bancos de material en Tlalpujahua se inició hace menos de 50 años, pero no ha sido hasta los últimos 5 años que la cantera vuelve a su auge comercial ya que en muchas ciudades del país se han unido elementos de la arquitectura colonia y conceptos modernos siendo el color rosa de los más solicitados.

En este trabajo se sometieron 3 distintos ejemplares de rocas ígneas a una solución preparada de sulfato de sodio (NMX-C-075-ONNCCE, 2006), simulando las condiciones de exposición a sales en ambientes marinos, intemperismo acelerado, provocando el deterioro prematuro y reduciendo la vida útil del elemento.

\section{PROCEDIMIENTO}

Las rocas de prueba fueron extraídas de distintos bancos de materiales. Las canteras Rosa (R) y Gris (G) del banco "LA AMÉRICA", mientras que la cantera Café Obscuro (Co) viene del banco "LLANO GRANDE” ambos ubicados en el municipio de Tlalpujahua, Michoacán, México.

Se cortaron 30 elementos cúbicos de $5 \mathrm{~cm} \times 5 \mathrm{~cm}$ de cada espécimen $\mathrm{R}, \mathrm{G}$ y $\mathrm{CO}$, cada cubo fue pesado, identificado y enumerado y se tomaron las medidas de longitud de cada una de sus caras.
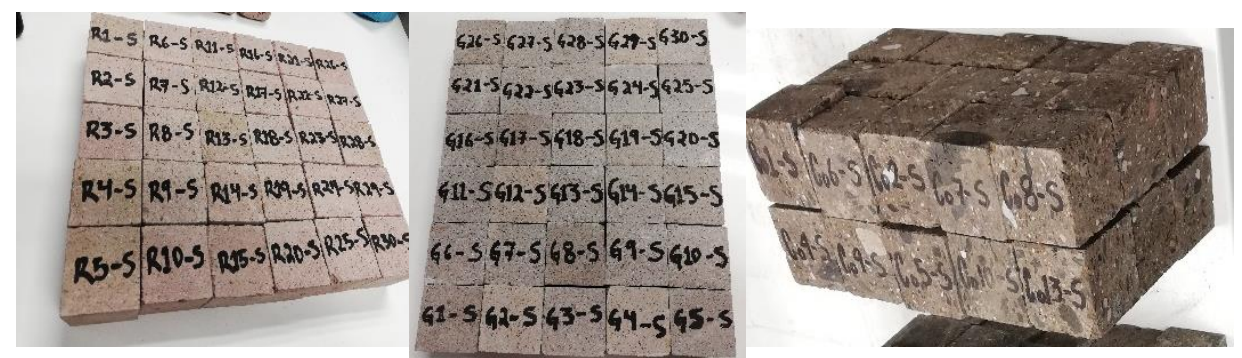

Figura 1. Cubos de Cantera que probar en exposición a sulfatos de sodio R, G y CO con su respectiva identificación.

\subsection{Preparación de la solución de Sulfato de sodio.}

Agregamos $1000 \mathrm{~g}$ de sal anhidra por cada 10 litros de agua, en total fueron usados 30 litros de agua (10L para cada espécimen) para la saturación a $22^{\circ} \mathrm{C}$, agregamos la sal lentamente al agua, mientras agitamos la mezcla por al menos 15 minutos o hasta que el agua se vuelva clara. Dejamos reposar la solución durante $48 \mathrm{~h}$ y rompemos la costra de cristales de sal que se forma en la superficie, agitamos una vez más y usamos.

\subsection{Pruebas previas a inmersión de las muestras.}

Antes de exponer las canteras a la solución de sulfato, estas fueron secadas en un horno hasta alcanzar una masa constante.

Sumergimos en agua potable hasta una completa saturación. Medimos sus dimensiones, su masa húmeda, masa sumergida y realizamos pruebas de Resistividad eléctrica (RE), Velocidad de pulso ultrasónico (VPU), Densidad, y a los primeros 5 cubos de cada color de cantera les hicimos la prueba 
de compresión simple. Estas primeras pruebas fueron registradas como ciclo 0. y fueron hechas con la finalidad de tener un punto de comparación entre una roca sana y el sucesivo desgaste de las que serían expuestas a la solución de sulfatos. Al finalizar 25 rocas restantes de cada color (en total 75 cubos) fueron colocados en el horno.

\subsection{Inmersión de las muestras en la solución.}

En unos recipientes plásticos vaciamos la solución de sulfato de sodio y colocamos una malla de plástico para que las rocas no estuvieran en contacto directo con la sal. Sacamos las rocas del horno una vez tuvieron masa constante. Las sumergimos en la solución procurando que ésta cubriera los cubos por $1 \mathrm{~cm}$ y fueron dejadas durante 18 horas.

\subsection{Inicio de ciclos de pruebas.}

Pasadas las 18 horas de inmersión de las muestras, fueron sacadas y comenzamos el Ciclo 1 midiendo las dimensiones, masa húmeda de cada cubo, masa sumergida, densidad, RE, VPU, y al final probamos a compresión simple los especímenes del 6 al 10 de cada Roca (R, G y CO). Registramos cada dato y volvimos a meter los restantes 60 cubos al horno.

Se repite el proceso anterior hasta llegar al Ciclo 5 o las rocas ya no puedan ser sometidas a ninguna prueba debido a la pérdida de masa.

\subsubsection{Prueba de Medición de dimensiones de cubos.}

Con ayuda de un vernier medimos 1 cara de cada cubo y su altura, sacando de esa forma su volumen y verificando que efectivamente cada lado medía $5 \mathrm{~cm}$, en el ciclo 0 . Conforme pasaban los ciclos las rocas tenían una menor área superficial y los ángulos eran menos marcados, dificultando la exacta medición.

\subsubsection{Masa húmeda y masa sumergida.}

Al sacar las muestras de la solución lo primero que tomábamos era la masa húmeda con ayuda de una báscula electrónica para mayor precisión y eficiencia ("figura\#").

La masa sumergida la tomamos con ayuda de una báscula manual, una canastilla y un recipiente lleno de agua. Sujetamos la canastilla a la báscula y la sumergimos en el agua, tomamos su peso sumergido (Mc). Después pusimos el espécimen dentro de la canastilla sumergida y pesamos (Mt), restamos el peso de la canastilla al peso final y así obtenemos la masa sumergida.

$$
M s=M t-M c
$$

\subsubsection{Densidad.}

La prueba se realiza con la roca completamente saturada, se seca superficialmente y se registra su peso húmedo (Wh en gramos), llenamos un picnómetro con agua y colocamos el cubo dentro, el volumen desalojado de agua $\left(\mathrm{V}\right.$ en $\left.\mathrm{cm}^{3}\right)$ es recolectado en una probeta, los cálculos se realizan con la siguiente ecuación:

$$
D=\frac{W h}{V}
$$

Siendo:

$\mathrm{D}=$ Densidad de la muestra, $\mathrm{g} / \mathrm{cm}^{3}$ 


\subsubsection{Resistividad Eléctrica (RE).}

La resistividad eléctrica es una propiedad de cada material, y corresponde al recíproco de su conductividad su unidad de medida es el ohm-cm u ohm-m. Depende en gran proporción del grado de saturación de los poros del espécimen. Necesitamos un equipo capaz de medir resistividad eléctrica en suelos y materiales porosos, un par de placas metálicas y almohadillas absorbentes de agua. Marcamos las caras donde se colocarían las placas metálicas (y posteriormente se haría la prueba a compresión simple). Humedecimos las almohadillas y se coloca la muestra. Se somete a la muestra a una corriente (I) y registrando un voltaje (E), se obtuvo una resistencia eléctrica dada en $\mathrm{k} \Omega$ usando la siguiente fórmula:

$$
\rho=\operatorname{Re}\left(\frac{A}{L}\right)
$$

Donde:

$\rho=$ Resistividad eléctrica, en $\mathrm{k} \Omega$-cm.

$\mathrm{Re}=$ Resistencia eléctrica, en $\mathrm{k} \Omega$.

$\mathrm{A}=$ Área transversal del espécimen, en $\mathrm{cm}^{2}$.

$\mathrm{L}=$ Longitud del espécimen, en $\mathrm{m}$.

\subsubsection{Velocidad de Pulso Ultrasónico (VPU).}

La prueba de velocidad de pulso ultrasónico es una prueba no destructiva conocida desde hace mucho tiempo y se considera como la relación que existe entre la distancia de viaje a través del espécimen de una onda ultrasónica y el tiempo que tarda en recorrerla. Este método es aplicable para evaluar la calidad de la muestra, y ayuda a identificar la porosidad del material y en el estudio para estimar la formación de grietas o en este caso el deterioro producido por la exposición agresiva a los sulfatos. Se requirió un equipo de velocidad de pulso ultrasónico. El espécimen debe tener las superficies planas y lisas, deben estar completamente saturado al momento de la prueba. Calibramos el aparato de velocidad de pulso a 2 pulsaciones por segundo e introducimos la altura de la muestra a probar. En los 2 transductores del aparato colocamos una fina capa de gel (esto sirve en caso de que la superficie no esté completamente lisa) y se coloca la roca entre los transductores firmemente, y se inician las pulsaciones. La prueba se finaliza cuando se obtiene un valor constante o estable. Tomamos como punto de comparación la información dada en la "Tabla 1" ya que en México no existe un criterio específico para rocas en VPU.

Tabla 1. Criterios de VPU con base a la norma NMX-C-530-ONNCCE-2017

\begin{tabular}{|c|c|}
\hline Velocidad de pulso Ultrasónico $(\mathbf{m} / \mathbf{s})$ & Clasificación \\
\hline$>4575$ & EXELENTE \\
\hline $4575>\mathrm{V}>3660$ & BUENO \\
\hline $3660>\mathrm{V}>3050$ & CUESTIONABLE \\
\hline $3050>\mathrm{V}>2135$ & POBRE \\
\hline $\mathrm{V}<2135$ & MUY POBRE \\
\hline
\end{tabular}

\subsubsection{Resistencia a la Compresión simple.}

La resistencia a compresión está definida como la capacidad de carga a compresión por unidad de área de la roca en el caso de esta investigación, cuya medida se da en ensayes de especímenes cúbicos, generalmente expresada en $\mathrm{kg} / \mathrm{cm} 2$. Y a diferencia de las pruebas anteriores es una prueba destructiva. Se necesitó una máquina universal de pruebas, donde colocamos cada uno de los cubos aprobar la base de esta y en el centro de la platina móvil para lograr una carga uniformemente distribuida sobre toda el área del cubo.

Se procedió a la aplicación de la carga a una velocidad constante hasta lograr la falla del cubo. 


$$
R=\frac{W}{A}
$$

Donde:

$\mathrm{R}=$ Resistencia a la compresión, en $\mathrm{kg} / \mathrm{cm}^{2}$.

$\mathrm{W}=$ Carga de ruptura de la muestra, $\mathrm{kg}$.

$\mathrm{A}=$ Área de la cara de prueba de la muestra, en $\mathrm{cm}^{2}$.

\section{RESULTADOS}

\subsection{Pérdida de masa}

Del ciclo 0 al 1 no se muestra una gran pérdida de masas, en el ciclo 2 y 3 se presenta un incremento de la masa debido a que la roca absorbió parte de la solución y se forman cristales de sal entre los poros de esta, no obstante durante el ciclo 4 la roca Co tuvo una gran pérdida de masa en comparación con las otras dos, siendo en el ciclo 5 la de mayor pérdida con $20.73 \mathrm{~g}$ de pérdida, y la $\mathrm{R}$ la de menor pérdida de las 3 con tan solo $6.42 \mathrm{~g}$ de reducción de masa.
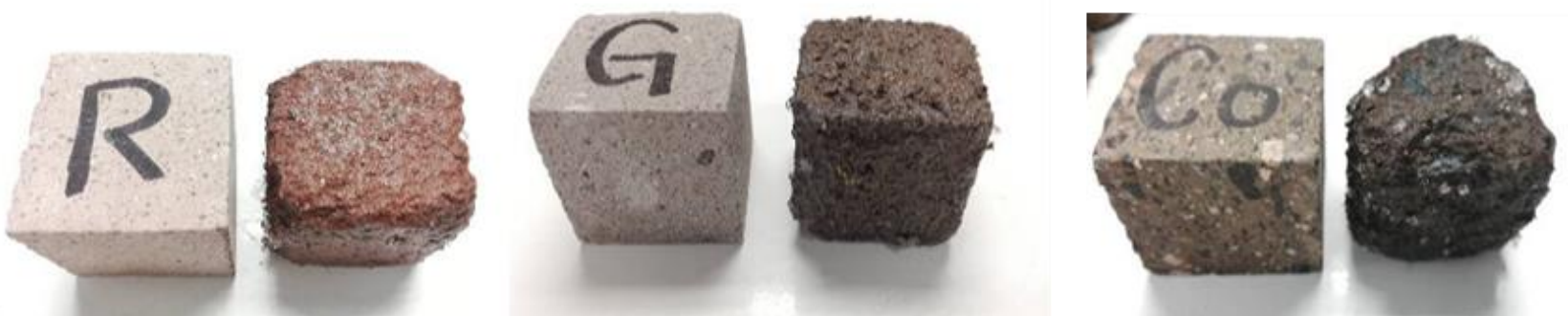

Figura2. Comparativa de canteras antes y después de la inmersión en la solución de sulfatos de sodio.

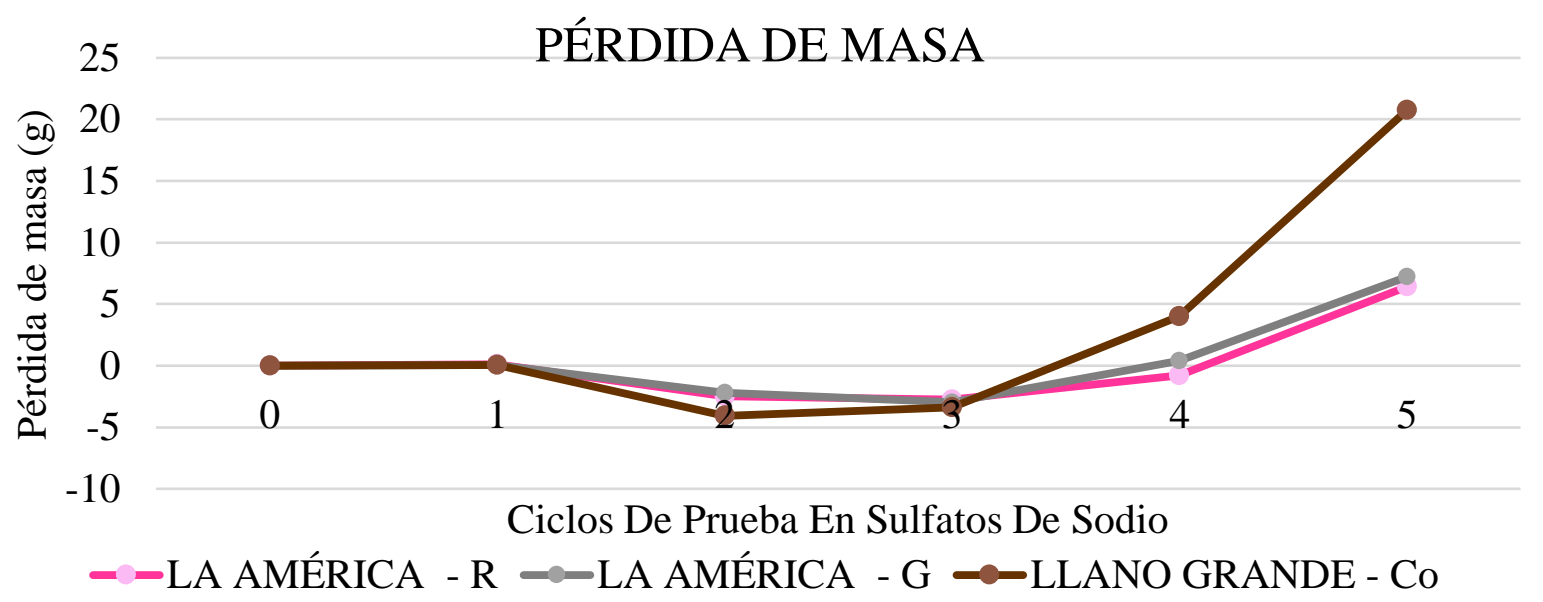

Figura 3. Resultados de pérdida de masa tomados al momento de sacar las rocas de su inmersión en la solución de sulfatos de sodio.

\subsection{Densidad}

AL inicio de las pruebas las rocas presentaban una densidad media (siendo el rango entre 2 y $4 \mathrm{~g} / \mathrm{cm}^{3}$ ), en el ciclo 3 la densidad de G llegó al rango de densidad baja, no obstante, en el último ciclo todas tuvieron un aumento en ella siendo la roca Co la mayor. Este aumento debido a la formación de cristales de sales de sulfato de sodio en los porosos de las rocas probadas, siendo la roca Co la más porosa y la roca rosa la de menor porosidad. 


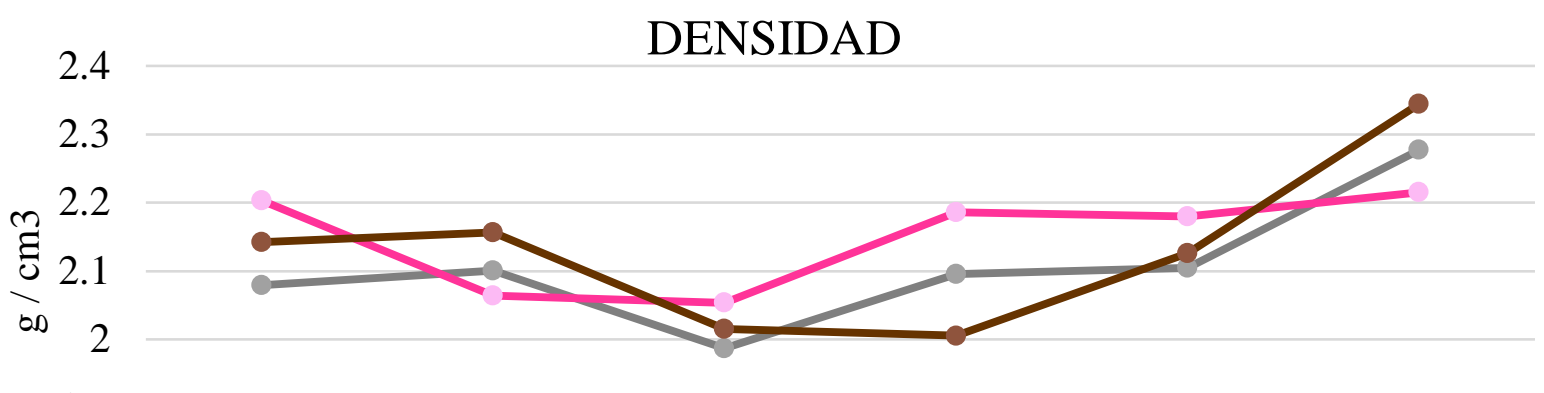

1.9

1.8

$\begin{array}{lcccc}0 & 1 & 2 & 3 & 4 \\ & \text { CICLOS DE PRUEBA EN SULFATOS DE SODIO } & 5\end{array}$

-LA AMÉRICA - G - -LA AMÉRICA - R —-LLANO GRANDE - Co

Figura 4. Densidades de roca Gris, Rosa y Café obscuro durante 5 ciclos.

\subsection{Resistividad Eléctrica (RE)}

Durante el ciclo 0 se mostraron buenos resultados ante la RE, no obstante, apenas fueron expuestas al sulfato (ciclo 1) hubo un cambio repentino teniendo resistividades menores a $2 \mathrm{kohms}-\mathrm{cm}$. Siendo la roca Co la que mejores resultados obtuvo. En el ciclo cero la resistividad ya era inexistente.

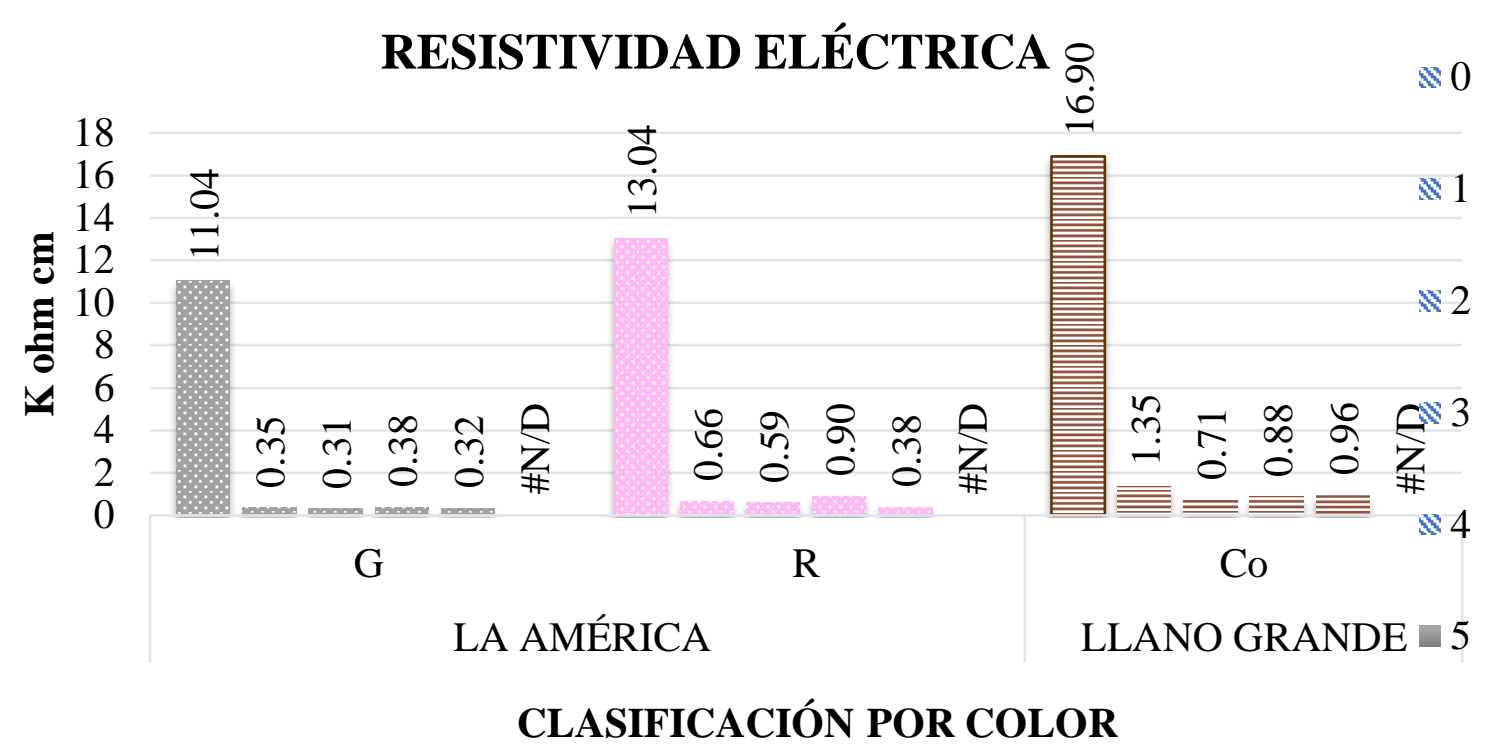

Figura 5. Resultado de resistividad eléctrica de cada roca durante 5 ciclos.

Tabla 1. Resultados de resistividad eléctrica por ciclo.

\begin{tabular}{|c|c|c|c|c|c|c|}
\hline $\begin{array}{c}\text { Promedio de } \\
\text { R.E. .-cm }\end{array}$ & \multicolumn{7}{|c|}{ Ciclos } \\
\hline $\begin{array}{c}\text { Banco y } \\
\text { Cantera }\end{array}$ & $\mathbf{0}$ & $\mathbf{1}$ & $\mathbf{2}$ & $\mathbf{3}$ & $\mathbf{4}$ & $\mathbf{5}$ \\
\hline G & 11.0360 & 0.3548 & 0.3112 & 0.3800 & 0.3248 & 0 \\
\hline R & 13.0412 & 0.6556 & 0.5893 & 0.9031 & 0.3805 & 0 \\
\hline Co & 16.8951 & 1.3535 & 0.7118 & 0.8786 & 0.9568 & 0 \\
\hline
\end{tabular}




\subsection{Velocidad de Pulso Ultrasónico}

La roca Co fue la que obtuvo más altos valores en esta prueba iniciando en el ciclo 0 con una VPU de 2875 , no obstante, en el último ciclo el área era tan irregular que fue imposible hacer la prueba. La roca rosa fue la que menor cambio tuvo conforme pasaban los ciclos. Mientras que la roca $\mathrm{G}$ fue muy similar a la rosa en todos los ciclos.

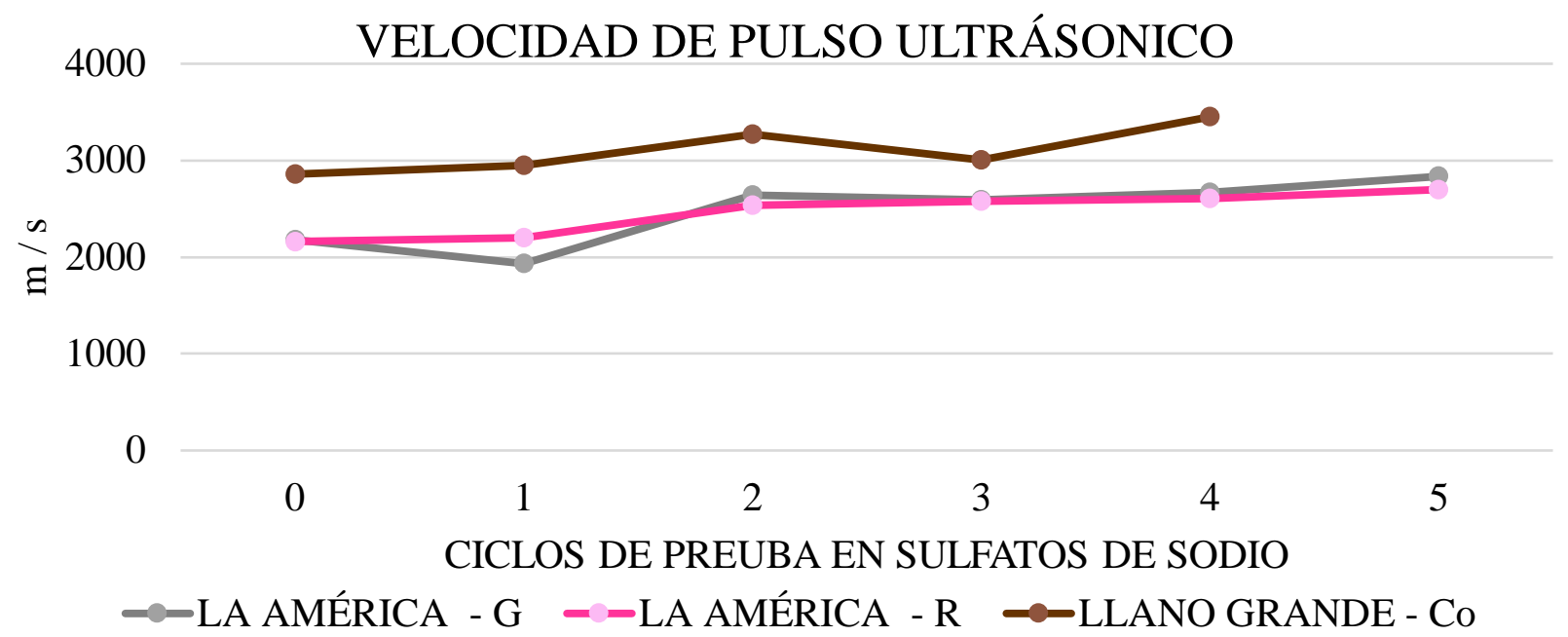

Figura 6. Comparativa gráfica de resultados en la prueba no destructiva de Velocidad de Pulso Ultrasónico en las rocas Co, G y R.

Tabla 2. Resultados de Velocidad de pulso ultrasónico en cada ciclo de cada roca probada.

\begin{tabular}{|c|c|c|c|c|c|c|}
\hline $\begin{array}{c}\text { Promedio de } \\
\text { VPU m/s }\end{array}$ & \multicolumn{7}{|c|}{ Ciclos de prueba } \\
\hline Rocas & 0 & 1 & 2 & 3 & 4 & 5 \\
\hline G & 2178.733333 & 1932.24 & 2638.45 & 2592.866667 & 2665.75 & 2834.5 \\
\hline R & 2161.266667 & 2197.92 & 2538.5 & 2578.666667 & 2602.9 & 2698.25 \\
\hline Co & 2857.8 & 2950.84 & 3267.15 & 3007.466667 & 3450.35 & $\#_{i}$ DIV/0! \\
\hline
\end{tabular}

\subsection{Resistencia a la compresión simple}

$\mathrm{Al}$ inicio de la prueba durante el ciclo 0 la roca que mostró mejor resistencia fue la roca $\mathrm{R}$ con un valor de $250 \mathrm{Kg} / \mathrm{cm}^{2}$, mientras que la Gris y la Co tuvieron una resistencia similar de entre $170 \mathrm{a} 180 \mathrm{Kg} / \mathrm{cm}^{2}$. Durante el segundo ciclo la rosa tuvo un pico de resistencia de casi $360 \mathrm{~kg} / \mathrm{cm}^{2}$ esto debido a la formación interna de cristales. Y en el último ciclo llegó a ser muy similar a la roca Gris. La roca Gris se mantuvo constante estando en el ciclo 0 en $182 \mathrm{~kg} / \mathrm{cm} 2$ y en el ciclo 5 a $132 \mathrm{~kg} / \mathrm{cm} 2$. La roca Co no pudo ser probada en el último ciclo Compresión simple debido a que la pérdida de masa imposibilitaba dicha acción. 


\section{RESISTENCIA MÁXIMA A COMPRESIÓN}

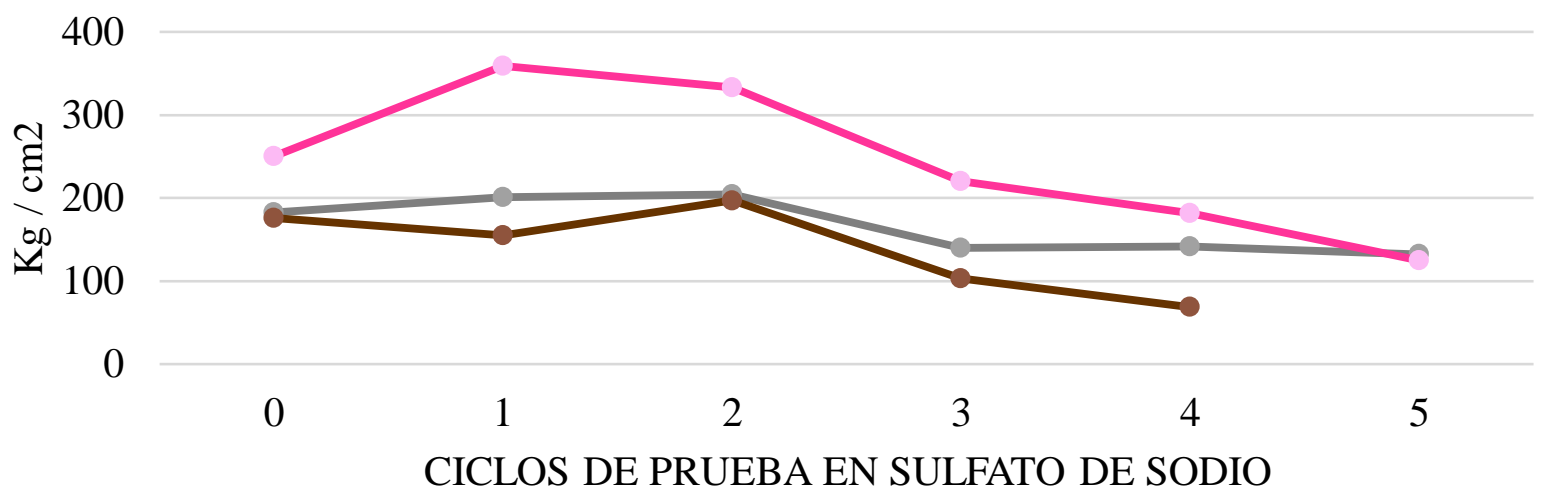

—-LA AMÉRICA - G --LA AMÉRICA - R —-LLANO GRANDE - Co

Figura 7. Comparativa de resistencia a la compresión directa de las rocas sumergidas en solución de sulfatos de sodios

Tabla 3. Resultados de las pruebas destructiva de Resistencia a la Compresión.

\begin{tabular}{|c|c|c|c|}
\hline $\begin{array}{c}\text { Promedio de } \\
\text { Compresión }\end{array}$ & \multicolumn{3}{|c|}{ Rocas } \\
\hline & LA AMÉRICA & & LLANO GRANDE \\
\hline ciclos & G & R & Co \\
\hline $\mathbf{0}$ & 182.9553589 & 250.1560547 & 175.8222913 \\
\hline $\mathbf{1}$ & 200.7636876 & 358.9915682 & 155.2235102 \\
\hline $\mathbf{2}$ & 204.2850147 & 333.3822086 & 197.1980928 \\
\hline $\mathbf{3}$ & 139.8297682 & 219.8915876 & 102.8854566 \\
\hline $\mathbf{4}$ & 142.0024707 & 182.1475148 & 68.5711153 \\
\hline $\mathbf{5}$ & 132.0291078 & 124.8476753 & \\
\hline
\end{tabular}

\section{CONCLUSIONES}

\subsection{Roca Café Obscuro (Co)}

La roca Café obscuro (Co) comparada a las otras dos presentó una mayor pérdida debido a que tenía una mayor porosidad y menor resistencia, esto complicó la realización de las otras pruebas no destructivas y destructivas, sin embargo en la prueba de VPU fue la que mejores resultados obtuvo, siendo "cuestionable" Según la (tabla 1) la cual pertenece a la normativa que rige las pruebas a concretos ya que no existe una norma que rija tan cual las pruebas a rocas, y se usan entonces como punto de comparación solamente.

\subsection{Roca Rosa}

La roca rosa fue la que mejores resultados obtuvo en resistencia a la compresión, pudiéndosele dar un uso estructural mayor que cualquiera de las otras dos. y se mantuvo más constante en la velocidad de 'pulso. Esto indica que la porosidad era menor en comparación con las otras dos rocas. Además, que fue la que menor pérdida de masa tuvo en los cinco ciclos. 


\subsection{Roca Gris}

Fue la que obtuvo resultados más constantes en todas las pruebas.

Se cumplió el objetivo de esta investigación, el cual era saber si la utilización de estos materiales era apta para los monumentos u otros edificios, ya fuera en restauraciones o en nuevas construcciones. Siempre tomando en cuenta que su uso principal es la ornamental. Y de esta forma conservar la arquitectura característica de nuestro país siendo patrimonio de México y en algunos casos de la humanidad.

\section{AGRADECIMIENTOS}

Agradezco al laboratorio de materiales "Ing. Luis Silva Ruelas" de la Facultad de Ingeniería Civil perteneciente a la Universidad Michoacana de San Nicolás de Hidalgo, por su apoyo tanto en material, equipo y el espacio de trabajo.

A José María García Perdomo por apoyarme con la adquisición de las rocas que fueron probadas.

A los bancos de materiales "La américa" y "Llano Grande" por su generosa donación de las canteras que fueron usadas durante esta investigación.

A la maestra Sandra y el Ingeniero Noel por su continuo apoyo, tanto en conocimiento como en apoyo práctico.

A los coautores de este artículo con quienes trabajé durante el desarrollo de este trabajo.

\section{REFERENCIAS}

Organismo Nacional de Normalización y Certificación de la Construcción y Edificación. (2004). NMXC-075-ONNCCE-2017 Industria de la Construcción-Agregados-Determinación de la Sanidad por Medio de Sulfato de Sodio o Sulfato de Magnesio-Método de Ensayo.ONNCCE.

Durar, R. (2000). Manual de Inspección, Evaluación y Diagnóstico de Corrosión en Estructuras de Hormigón Armado (Tercera ed.). México.

Organismo Nacional de Normalización y Certificación de la Construcción y Edificación. (2004). NMXC-073-ONNCCE-2004: Método de prueba estándar para la masa unitaria en agregados. ONNCCE.

Organismo Nacional de Normalización y Certificación de la Construcción y Edificación. (2004). NMXC-275-ONNCCE-2004: Método de prueba estándar para la velocidad de pulso a través del concreto. ONNCCE.

Organismo Nacional de Normalización y Certificación de la Construcción y Edificación. (2014). NMXC-083-ONNCCE-2014: Método de prueba estándar para la resistencia a la compresión de especímenes. ONNCCE.

Organismo Nacional de Normalización y Certificación de la Construcción y la Edificación. (2004). NMX-C-030-ONNCCE-2004: Práctica estándar para el muestreo de agregados. ONNCCE. https://www.mexicodesconocido.com.mx/la-cantera-de-tlalpujahua-estado-de-mexico.html. México desconocido. La cantera en tlalpujahua, 30 de mayo 2019.

Manual de Análisis de Materiales. Laboratorio de Materiales ingLuis Silva Ruelas. Tercera Edición. México 2011. 